10

\title{
Endocrine-disrupting chemicals and their effects on puberty
}

\author{
David Lopez-Rodriguez, PhD in Psychology and Research \\ Scientist ${ }^{\mathrm{a}}$, \\ Delphine Franssen, PhD in Biomedical Science and Research \\ Scientist ${ }^{\mathrm{a}}$, \\ Sabine Heger, Pediatric Endocrinologist, Associate \\ Professor ${ }^{\mathrm{b}, * *, 1}$, \\ Anne-Simone Parent, Pediatric Endocrinologist, Associate \\ Professor and Research Group Leader ${ }^{\mathrm{a}, \mathrm{c}, *, 1}$ \\ a GIGA Neurosciences, Neuroendocrinology Unit, University of Liège, Belgium \\ ${ }^{\mathrm{b}}$ Children's Hospital Bult, Janusz-Korczak-Allee 12, 30173, Hannover, Germany \\ ${ }^{\mathrm{c}}$ Department of Pediatrics, University Hospital Liège, Belgium
}

\section{A R T I C L E I N F O}

Article history:

Available online 17 September 2021

Keywords:

pubertal timing

secular trend

hypothalamus

GnRH

epigenetics

\begin{abstract}
Sexual maturation in humans is characterized by a unique individual variability. Pubertal onset is a highly heritable polygenic trait but it is also affected by environmental factors such as obesity or endocrine disrupting chemicals. The last 30 years have been marked by a constant secular trend toward earlier age at onset of puberty in girls and boys around the world. More recent data, although more disputed, suggest an increased incidence in idiopathic central precocious puberty. Such trends point to a role for environmental factors in pubertal changes. Animal data suggest that the GnRH-neuronal network is highly sensitive to endocrine disruption during development. This review focuses on the most recent data regarding secular trend in pubertal timing as well as potential new epigenetic mechanisms explaining the developmental and transgenerational effects of endocrine disrupting chemicals on pubertal timing.
\end{abstract}

(c) 2021 Elsevier Ltd. All rights reserved.

\footnotetext{
* Corresponding author. GIGA Neurosciences, Neuroendocrinology Unit, University of Liège, Belgium.

** Corresponding author.

E-mail address: asparent@uliege.be (A.-S. Parent).

1 Equal contribution.
} 


\section{Introduction}

Puberty represents a crucial developmental milestone leading to attainment of reproductive function and allowing species survival. It is controlled by a tightly organized network of neurotransmitters, neuropeptides as well as (epi-)genetic and hormonal factors, which ultimately drive the pulsatile secretion of Gonadotropin-Releasing Hormone (GnRH). Such organizational processes take place during fetal and early postnatal life. Therefore, exposure to environmental pollutants such as Endocrine Disrupting Chemicals (EDCs) during critical periods of development might affect pubertal timing and long-term reproductive consequences. Clinical data supporting the existence of a secular trend in pubertal onset and incidence in idiopathic central precocious puberty will be reviewed. Such trends suggest a role for environmental factors including EDCs. While a substantial body of evidence shows that the gonads and peripheral organs are targets of EDCs, recent animal data underline their impact on the developing brain and the hypothalamic control of puberty. In particular, this review will focus on newly described epigenetic mechanisms and their consequences on puberty and reproduction throughout generations.

\section{Neuroendocrine regulation of the HPG-Axis at puberty}

The key event of initiation of pubertal development is the gonadal steroid independent increase of pulsatile gonadotropin-releasing hormone (GnRH) from GnRH neurons located in the hypothalamus $[1,2]$. GnRH is secreted into the pituitary portal veins, subsequently stimulating the episodic systemic secretion of luteinizing hormone (LH) and follicle stimulating hormone (FSH) from the anterior pituitary gland [3]. These two hormones coordinate the function of the gonads, enabling them to produce sex steroids and gametes. GnRH secretion is not only activated at the advent of puberty, in fact GnRH release already occurs during fetal development and postnatally during "mini-puberty" [4,5]. The pubertal increase in $\mathrm{GnRH}$ secretion is determined by changes in transsynaptic [2,6] and glial [7] inputs to the GnRH neuronal network. In addition, recent evidence suggests that epigenetic mechanisms serve as an essential coordinator of the modulation of the pubertal process [8].

Neuroendocrine mechanisms controlling GnRH secretion are established during late gestation and "mini-puberty". The GnRH neurosecretory system is essentially regulated by kisspeptin neurons during these developmental periods, which are followed by central inhibition during childhood [2]. Recent work strongly suggests that in addition to the major inhibitory neurotransmitter gamma-aminobutyric acid (GABA) [9] macorin 3 (MKRN3) serves as a kisspeptin inhibiting signal. MKRN3 selectively inhibits KISS1 and TAC3 promoter activity, indicating an indirect upstream inhibition of GnRH [10]. MKRN3 itself is down regulated by hypothalamic microRNA, miR-30b, whose expression increases during postnatal maturation [11]. Interestingly, GABA inhibition on $\mathrm{GnRH}$ release occurs also at the kisspeptin signalling level $[2,12]$.

In humans and other primates, most $\mathrm{GnRH}$ cell bodies are located in the medial basal hypothalamus $(\mathrm{MBH})$, the infundibulum and periventricular regions $[13,14]$. They originate from the olfactory placode migrating to their final destination during development $[15,16]$, a process that is regulated by many factors [17]. Some of them have been identified while searching for molecular aetiologies for delayed or precocious puberty [18-20]. Surrounding cells, such as glia make up to fifty percent of brain cells in humans, primates and rodents [21] and regulate GnRH neuronal activity by acting at the level of the cell bodies and nerve endings. Through these mechanisms they essentially contribute to the hypothalamus-pituitary-gonadal (HPG) integration of endocrine, metabolic and environmental factors to regulate reproductive function. As part of the blood-brain barrier, they integrate circulating metabolic signals such as sirtuins [22], AMPK [23,24] and other endocrine signals.

Because of its phases of activity and inactivity, its special developmental features and its close interchanges with other delicately regulated systems, the GnRH-neuronal network offers several targets for endocrine disrupting mechanisms. 


\section{Secular trend in pubertal timing and incidence of precocious puberty}

Pubertal onset in girls is marked by breast development which takes place between age 8 and 13.5 years followed by pubic hair development and increased growth velocity. The end of puberty in girls corresponds to menarche followed by ovulatory cycles several months later. In boys, puberty onset is marked by genital development taking place between age 9 and 14 years with testicular volume reaching a volume above $4 \mathrm{ml}$. It is followed by pubic hair development and increased growth velocity. The end of puberty corresponds to a testicular volume of $15-20 \mathrm{ml}$. Pubertal timing in humans is characterized by a unique individual variability. The physiological range of age at onset of puberty covers 5 years which represents about $6 \%$ of a life span [25]. Individual variance is much lower in other well described models such as rodents, sheep or birds [25]. Only some non-human primates show a variance of pubertal timing that is even longer than humans and represents $10 \%$ of average life span [26]. While genetic factors are considered to be the major determinant of age at puberty, nutrition, socioeconomical factors and environmental pollutants affect pubertal onset as well [25].

Historically, secular trend in pubertal timing has been established based on reports about age at menarche. Menarche was indeed the most accurate and easy to remember clinical sign of pubertal development. However, time has shown that the different pubertal endpoints follow different trends. Age at menarche has been reported to decrease between 1890 and 1960 in the USA and most European countries, consistently with improvement in nutritional status and socioeconomic conditions [25]. Age at menarche appears to have stabilized since the sixties in those countries. However, the USA and Denmark have reported a slight decrease in more recent studies between 2002 and 2008 [27,28] with a median age at menarche being 12.2 in the American study and 13.1 in the Danish study. Notably, age at menarche is still decreasing in emerging countries such as India, China, Nigeria [29] or some European countries such as Romania [30]. Age at menarche remains different between girls from higher and lower socioeconomical status or urban and rural origin in emerging countries while Romania has documented a disappearance of such difference [30].

While age at menarche appears to overall stabilize in the Western world, age at thelarche continues to decrease. A recent systematic review and meta-analysis showed that age at breast development defined by physical or clinical examination has decreased by a mean of almost 3 months per decade from 1977 to 2013 worldwide [31]. Secular trend in pubertal onset in girls is well documented but data in boys is scarce. Recent studies seem to indicate that age at peak height velocity in boys is decreasing. A Swedish study has recently reported that peak height velocity was 1.5 months earlier for every decade increase in birth year in a population of 4090 participants born between 1951 and 1996 [32]. Similarly, a Danish study showed that peak height velocity was 0.3 years earlier in boys born between 1965 and 1970 compared to boys born 1935-1939 [33]. These observations are of prime clinical relevance as the definition of normal age at onset of puberty will determine who undergoes exploration for precocious puberty. Additionally, such secular trends strongly suggest environmental influences among which the ongoing global obesity epidemics [34] and endocrine disrupting chemicals [25] have been identified.

Although data is limited, it appears that the number of girls addressed for suspicion of precocious puberty is also increasing, again suggesting the existence of environmental factors. While some studies based on medication prescription and reimbursement suggest an increase in central precocious puberty in Europe [35-37], incidence of premature thelarche and premature adrenarche is almost never documented. However, a recent Danish study showed a substantial increase of central precocious puberty, premature thelarche and premature adrenarche in Danish girls between 1998 and 2017 [38]. The trend was even stronger in first and second-generation immigrants. Notably, incidence of central precocious puberty in boys had strongly increased as well but this trend was not confirmed in a shorter and smaller American study [39].

\section{Pubertal timing and EDC}

Several studies have reported relations between early (prenatal or childhood) exposure to EDCs and clinical timing of puberty [40] or concentrations of circulating reproductive hormones [41], indicating alterations of the development of the hypothalamic-pituitary-gonadal axis. A recent review indicated 
that prenatal or childhood exposure to non-persistent pesticides is associated with altered pubertal timing in boys and girls [42].

Data regarding effects of EDCs on pubertal timing in humans is often contradictory due to the difficulties of such studies. Girls and boys are exposed to low doses of hundreds of chemicals, rendering causation difficult to demonstrate. Most studies focus on a single family of compounds. Consistently with the developmental origin of adult health, exposure may have taken place during early development leading to a long latency between exposure and the potential consequences on pubertal timing. However, most studies are retrospective or cross-sectional. Moreover, the effects of exposure to endocrine disruptors can differ depending on the studied endpoint (i.e. breast development or menarche). For these reasons, animal studies are very important to determine causal relationships and involved mechanisms (neuroendocrine versus peripheral mechanisms).

\section{Crucial developmental time periods and EDC exposure}

Developing organisms are particularly sensitive to EDC exposure. Fetal growth relies on maternal nutritional intake and oxygen [43] and is regulated by a complex interaction between maternal, placental and fetal factors [44]. The physiological mechanisms regulating fetal growth are sensitive to maternal exposure to drugs, toxins, alcohol but also EDCs. Several EDCs cross the placental barrier via passive or active transport [45,46]. Consequently, Bisphenol A, phthalates, pesticides and others EDCs have been detected in the placenta and amniotic fluid [47-49] and have been linked to impairment of fetal development [50,51]. It is considered that each fetus has been exposed to a hundred chemicals before birth [52].

In regard to the reproductive axis, crucial processes such as the migration of GnRH neurons and their integration into the hypothalamic circuitry take place during the fetal period to assure a tight regulation of pubertal timing and later reproduction. GnRH neurons migrate from the olfactory placode to reach the hypothalamus between embryonic day 17 and 18 in rats [15,53]. Alteration of this migration results in delayed puberty or Kallman syndrome in humans [54]. Early exposure to some EDCs seems to affect this crucial process. Studies in zebrafisch emphasize, that exposure of zebrafish embryos to $17 \alpha$-ethinylestradiol (EE2) or nonylphenol (NP) disrupts the ontogeny of GnRH neurons in the forebrain via ER pathway [55,56]. The number of GnRH expressing neurons is increased due to exposure to EE2 or NP during development. This effect is blocked by the ERs antagonist ICI 182-780 [56]. Similar observations have been reported in mice by Pillon et al. They demonstrated that exposure to EE2 at environmental doses of $0.01,0.1$ or $1 \mu \mathrm{g} / \mathrm{kg} /$ day between embryonic day E10.5 and E13.5 induces a dose-dependent increase in the total number of GnRH neurons at E13.5. Interestingly the distribution of GnRH neurons along the migratory path is not disrupted [57]. This effect is associated with limited but significant growth retardation. BPA $(2 \mu \mathrm{g} / \mathrm{kg} / \mathrm{d})$ exposure from E10 to P7 also appears to affect GnRH neuron development although it decreases the number of GnRH expressing cells in pubertal male rats [58]. This reduction of GnRH cells was observed after birth, thus it is not determined whether BPA exposure impairs GnRH neuron survival or migration.

As pointed out above, the neuroendocrine regulation of puberty involves the interaction of kisspeptin neurons within the GnRH network. Kisspeptin neurons are major activators of GnRH neurons during puberty and constitute two hypothalamic populations. In rodents, one is located in the arcuate nucleus (ARC) and appears between E11.5 and E13.5 in mouse and one is located the AVPV for which the precise timing of development is less clear but it is supposed to be between P10 and onset of puberty in rodents [59]. These neuronal populations are sensitive to estrogen. AVPV kisspeptin neurons mediate the estradiol positive feedback in adult animals while the ARC population is negatively regulated by estradiol and plays a role in pubertal onset [60]. Kisspeptin neurons seem to be sensitive to early exposure to EDCs. Recently, Pino et al. showed that exposure to BPA ( $5 \mu \mathrm{g} / \mathrm{kg} / \mathrm{d}$ ) from E11 to P8 alters the developmental maturation of kisspeptin neurons in female mice. Mice exposed to low doses of BPA from E11 to P8 exhibit a persistent, but divergent, impairment of kisspeptin neuronal maturation, resulting in a higher number of kisspeptin neurons in the AVPV but consistently fewer 
kisspeptin neurons and lower Kiss1 and Tac2 expression in the ARC. These changes in kisspeptin populations were associated with phenotypic signs of early puberty (early vaginal opening) and with suppression of circulating LH levels. Additional studies reporting EDC impact on kisspeptin neurons have been recently reviewed in Lopez-Rodriguez et al., 2021.

Pubertal maturation also requires normal gonadal development that takes place early in fetal life. During early embryonic development, human male and female gonads or germ cells are morphologically identical. Their differentiation into testes or ovaries is under the regulation of a complex genetic cascade [61] and hormonal input [62]. Germ cells are sensitive to exogenous perturbation. For example, exposure to paracetamol from E7 to E13.5 induces a reduction of the number of germ cells in female mouse fetus while exposure from E7 to birth leads to a decrease in ovarian follicle reserve and leads to subfertility in adulthood [63]. A delay in prophase I phase has also been recorded after exposure to BPA [64] and Di(2-ethylhexyl) phthalate DEHP [65]. The consequences of EDC exposure on gonadal development have been reviewed in depth in Johansson et al. [66].

Another critical developmental window for EDC exposure is the time of puberty itself. Reactivation of the hypothalamic-pituitary-gonadal axis and gonadal maturation are under the control of a tight neuronal network that is sensitive to exogenous perturbation. Several studies have shown pubertal precocity or delay in rodents after EDC exposure during puberty [25]. The limited number of mechanistic studies appears to point at kisspeptin as a potential target again. Pubertal exposure (P26-P30) to dibutyl phthalate $(0.5 \mathrm{mg} / \mathrm{kg})$ in female rats leads to increased $\mathrm{ER} \alpha$, Kiss1R and Kiss1 mRNA expression in the AVPV but a decrease in kisspeptin immunoreactivity in the ARC. These changes are associated with early puberty and higher serum estradiol levels [67]. Similar results were observed after prepubertal exposure (P15 to P20) to Zearalenone (5 mg/kg), an oestrogenic mycotoxin, which induces early vaginal opening, elevated LH, FSH and estradiol serum levels and increased GnRH, Kiss1 and Kiss1R mRNA and protein expression in the hypothalamus of female rats [68]. In female rhesus monkeys, direct BPA ( $10 \mathrm{nM})$ infusion into the median eminence from mid to late puberty suppresses GnRH and kisspeptin release [69].

It seems that the pubertal period is a window of high ovarian vulnerability to EDCs. AhR signaling regulates E2 production in the ovary at the end of the prepubertal period as illustrated by AhR KO mice which show decreased E2 synthesis and expression of steroidogenesic factors exclusively in late juvenile mice [70]. Such mechanism is disrupted by EDC acting through AhR [71,72].

All together, these data indicate that the fetal and pubertal developmental windows are very sensitive to EDC exposure. Environmental disruption during these periods can compromise sexual maturation.

\section{Newly described mechanisms of action for EDCs and their possible role in the control of puberty}

Endocrine disrupting chemicals (EDC) alter physiological reproductive functions through several mechanisms. Many of them mimic or block endogenous hormone action and display estrogenic or antiandrogenic mechanisms. They can bind to estrogen receptors (ER alpha and beta), estrogen-related receptors (EERgamma), androgen receptors [72-74] and the xenobiotic receptor aryl hydrocarbon receptor [75-77]. They act via G protein-coupled receptors (GPR), GPR30, calcium-signaling and orphan receptors [78]. Thus they alter gene expression as well as intracellular transduction pathways [79]. After incorporation they can also compete with endogenous hormones and other substances for binding to carrier proteins [80] or metabolization and interfere with epithelial cells triggering systemic para-inflammation [81].

Neurodevelopmental toxicity to the developing brain is exerted by increased oxidative stress, altered neurotransmitter systems, immune system disruption and neuroendocrine disruption [82]. A substantial body of work has identified alterations of epigenetic mechanisms caused by EDC action [83]. This important mechanism seems to be responsible for transgenerational and multigenerational 
effects of EDC [84-86] as well as indirect consequences such as disruption of circadian rhythms [87] or metabolic systems $[88,89]$. Recent discoveries have shown that the transcriptional activation of genes critical for pubertal onset is mediated by epigenetic factors. While it is known that EDCs affect DNA organization, the mechanisms through which epigenetic information is conveyed from environmental disruptors to the hypothalamic cells controlling pubertal onset and ovulation remains to be deciphered.

Three different mechanisms have been identified as epigenetic processes affecting gene expression without modifying DNA sequence: DNA methylation, post-translational histone modifications and non-coding RNA. These processes have been found to be involved in the activation of the transcriptional machinery leading to the activation of GnRH neurons in the hypothalamus and to be vulnerable to EDC exposure (Fig. 1) [83,90-93].

DNA methylation requires the covalent addition of a methyl group catalysed by DNA methyltransferases (DNMTs) usually at the carbon-5 position of cytosines in CpG dinucleotides. DNA methylation allows the recruitment of methyl-binding proteins to the gene promoter region which leads to gene silencing and heterochromatin formation [94]. DNA methylation has been proven to affect gene expression in the brain (i.e. Esr1, Kiss1) and influence sexual differentiation and puberty onset [95-97]. Loss of DNA methylation at the GnRH promoter region during the juvenile period facilitates its transcription and the activation of the neuroendocrine system leading to puberty [97]. In pubertal boys and girls, urine EDC levels were found to be associated with specific changes in the epigenome [98]. For instance, children with higher levels of phthalates showed higher DNA methylation levels at the thyroid hormone receptor interactor 6 (Trip6) promoter region, a gene associated with pubertal onset [99]. In animal models, EDCs have been shown to alter DNA by affecting DNMT enzymatic activity or expression. In male and female rodents, developmental exposure to BPA alters the maintenance and de novo DNA methylation by altering Dnmt1 and Dnmt3a-b mRNA expression in the brain and gonadal tissue during the peripubertal period [100,101]. Dnmt1 and Dnmt3b alterations were found to persist until adulthood [102-104]. Furthermore, 2,3,7,8-tetrachlorodibenzo-p-dioxine (TCDD) but not BPA was found to selectively decrease Dnmt1, Dnmt3a and Dnmt3b levels in GnRH immortalized cells (GT1-7) [105]. Downregulation of Dnmt1 caused by BPA exposure at P28 was found to be associated with hypomethylation of the Esr1 gene promoter in the hypothalamus [100]. Similar alterations were associated with disrupted estrous cyclicity and advanced reproductive senescence after perinatal exposure to methoxychlor [106]. In male mice, methoxychlor and vinclozolin exposure led to hypo- and hypermethylation of imprinted genes in the testis [107]. These results suggest sexdependent alterations of global or gene-specific methylation across different hormone-dependent tissues after developmental exposure to EDCs.

Transcriptional regulation of gene expression by histone post-translational modifications (PTMs) involves chemical modifications (acetylation, methylation, ubiquination) at specific amino acids of the histone tail. Histone PTMs alter gene expression by modifying chromatin structure and allowing an accessible or repressive state. During puberty, the Kiss1 gene promoter region undergoes a change in its chromatin landscape leading to its transcriptional activation. This activation occurs through the replacement of the repressive H3K27me3 histone mark by H3K4me3 and H3K27Ac activatory marks and is mediated by the Polycomb and Trithorax groups of proteins, respectively [91]. While the epigenetic mechanisms through which EDCs interfere with the GnRH network are still unknown, studies have shown that EDCs alter PTMs in the brain. For instance, perinatal exposure to BPA increases the levels of histone deacetylase Hdac2 in the hypothalamus and cortex of P21 and adult male mice [104]. TCDD exposure inhibits the expression of histone-modifiers Hdac1, Hat1, and Mecp2 mRNA in GT1-7 cells by interacting through the AhR/XRE signaling pathway, and reduces GnRH mRNA levels [105].

MicroRNAs (miRNAs) are short non-coding RNAs known to be expressed in sexual dimorphic regions of the hypothalamus and to be sensitive to oestrogens [108]. miRNAs are involved in the transcriptional control of pubertal onset. By acting post-transcriptionally, miR-200, miR-155 and miR-30 downregulate gene expression of known GnRH repressors such as Zeb1, Cebpb and Mkrn3, allowing the 
Fig. 1. Direct, multi- and transgenerational effects of EDCs. In directly exposed gestating females (F0 generation), embryos are exposed in utero and through lactation (F1 generation). As the germline is generated during embryonic life, F2 generation are also directly exposed in a multigenerational fashion. Transgenerational inheritance happens in the F3 and subsequent generations. (a) Embryonic life (i.e. male and female germline differentiation), is a specific window of vulnerability to EDC exposure. The germline undergoes a wave of epigenetic reprogramming in a sexdependent manner. After full demethylation of the germline around E12.5, males quickly undergo prospermatogonial differentiation whereas the female germline remains in meiotic arrest until early postnatal life. EDC exposure during this period may alter selection process critical for gonadal development and the reproductive function. (b) Perinatal EDC exposure may interfere with developmental processes involved in the somatic programming of the brain. Alterations in GnRH neuron migration and development may have irreversible consequences on reproductive functions, later in life or in subsequent generations. 
transcriptional activation of the GnRH network at puberty [11,93]. While miR-200 is selectively enhanced in GnRH neurons, miR-155 appears to control upstream regulators of the GnRH network. Loss of function of these miRNAs leads to disruption of pubertal onset and failure to display normal estrous cyclicity [93]. Lin28/let-7 in the hypothalamus is another miRNA system necessary to pubertal onset in rats which is sensitive to neonatal exposure to estrogens or androgens [109]. Very few studies have focused on EDC effects on miRNA but they seem to indicate sexually dimorphic disruption. A short prenatal exposure to PCBs increases the expression of let-7a, miR-219, miR-132, miR-7 and $m i R-145$ in prepubertal female rats in the preoptic area [108] and decreases it in adult males. Alterations of miRNA and steroidogenesis have also been observed after BPA exposure in sheep and mice ovaries and testis $[110,111]$.

In summary, it appears that EDCs could target the epigenetic mechanisms regulating the activation of GnRH neurons or gonadal steroidogenesis around puberty.

\section{Multi- and transgenerational effects of EDCs on puberty}

Exposure to environmental stressors in utero increases the risk of disease in adulthood but also throughout generations (Fig. 1). Exposure during gestation (F0 generation) causes F1 offspring to be exposed in utero and F2 generation to be exposed through germ cells. This is known as multigenerational inheritance. Transgenerational inheritance occurs when the effects of exposure are transmitted to the F3 and subsequent generations, which are not directly exposed. The mechanism of transgenerational transmission involves the inheritance of epigenetic information through the germline via mitotically and meiotically heritable changes in gene function. In mammals, the germline goes through a wave of epigenetic reprogramming during migration into the genital ridges. Germline remethylation occurs at different developmental stages in a sex-specific manner. In rodents, male remethylation and prospermatogonial differentiation starts around E14.5 and is completed by E17. In females, the germline remains in mitotic arrest and differentiates later at P1-5, when initial follicular assembly and recruitment start. This may lead to sex differences in windows of sensibility to epigenetic effects of EDCs.

EDCs may interfere with germline reprogramming and differentiation and alter reproductive function across generations. Few studies have focused on transgenerational effects of EDCs on puberty. Gestational exposure (F0) to TCDD [112], a mixture of pesticides or plasticizers [113] induces early pubertal onset in $\mathrm{F} 3$ female rats, together with a decrease in ovarian primordial and prenatal follicles. In males, these pubertal alterations are accompanied by increased apoptotic spermatogenic cells. Differential DNA methylation is associated with exposure to TCDD and the mixture of plasticizers in the testis. BPA exposure has been shown to upregulate the IncRNA encoded by the maternally expressed gene Meg3, a gene associated with puberty timing [114] in the brain of F3 juvenile mice [115].

EDCs may indirectly impair the transcriptional control of gene expression by affecting epigenetic control regions (ECR) or by interfering with the chromatin landscape of the germline [84]. How epigenetic alterations in the germline impair the programming of specific somatic tissue such as the hypothalamic neurons regulating puberty still needs to be elucidated.

\section{Conclusion}

The last 30 years have shown a global decrease in age at onset of puberty in girls and boys although the later is less documented. This has led to an active debate regarding the definition of precocious puberty. Besides this highly relevant clinical question, secular trend in puberty could be the canary in the coal mine indicating effects of EDCs on the developing brain. Animal data point toward a high developmental sensitivity of the GnRH network to EDCs. In particular, more recent data have identified potential epigenetic mechanisms which coul explain the integration of environmental signals by the hypothalamus and the transmission of phenotypic traits and pathologies throughout generations. 


\section{Research agenda}

- Secular trend in precocious telarche and precocious puberty incidence needs to be further documented.

- Exploring the effects of EDCs on the crosstalk between the hypothalamic control of energy balance and reproduction is of prime importance.

- The effects of EDC mixture on pubertal development need to be studied in order to inform environmental agency decisions.

- Much remains to be done to identify the pathways conveying epigenetic information from environmental disruptors to the hypothalamic cells controlling pubertal onset and ovulation.

\section{Declaration of competing interest}

The authors have no conflict of interest to declare.

\section{Acknowledgements}

This project has been supported by the Fonds National de la Recherche Scientifique (Belgium), the Belgian Society for Pediatric Endocrinology and Diabetology and the "Fonds Léon Fredericq".

\section{References}

[1] Wildt L, Marshall G, Knobil E. Experimental induction of puberty in the infantile female rhesus monkey. Science 1980; 207:1373-5.

[2] Terasawa E, Garcia JP. Neuroendocrine mechanisms of puberty in non-human primates. Curr Opin Endocr Metab Res 2020;14:145-51.

[3] Grumbach MM. The neuroendocrinology of human puberty revisited. Horm Res 2002;57(Suppl 2):2-14.

[4] Grumbach MM. Commentary: a window of opportunity: the diagnosis of gonadotropin deficiency in the male infant. J Clin Endocrinol Metab 2005;90:3122-7.

[5] Bouvattier C, Maione L, Bouligand J, et al. Neonatal gonadotropin therapy in male congenital hypogonadotropic hypogonadism. Nat Rev Endocrinol 2012;8:172-82.

[6] Ojeda SR, Skinner MK. Chapter 38. Puberty in the rat. In: Knobil E, Neill JD, editors. Knobil and Neill's Physiology of reproduction, volumen 2. Gulf Professional Publishing; 2006. p. 2061-103.

[7] Prevot V, Dehouck B, Sharif A, et al. The versatile tanycyte: a hypothalamic integrator of reproduction and energy metabolism. Endocr Rev 2018;39:333-68.

*[8] Aylwin CF, Toro CA, Shirtcliff E, et al. Emerging genetic and epigenetic mechanisms underlying pubertal maturation in adolescence. J Res Adolesc 2019;29:54-79.

[9] Ojeda SR, Roth C, Mungenast A, et al. Neuroendocrine mechanisms controlling female puberty: new approaches, new concepts in International Journal of Andrology. Int J Androl 2006:256-63.

[10] Abreu AP, Toro CA, Song YB, et al. MKRN3 inhibits the reproductive axis through actions in kisspeptin-expressing neurons. J Clin Invest 2020;130:4486-500.

[11] Heras V, Sangiao-Alvarellos S, Manfredi-Lozano M, et al. Hypothalamic miR-30 regulates puberty onset via repression of the puberty-suppressing factor, Mkrn3. PLoS Biol 2019;17:e3000532.

[12] Bizzozzero-Hiriart M, Di Giorgio NP, Libertun C, et al. GABAergic input through GABAB receptors is necessary during a perinatal window to shape gene expression of factors critical to reproduction such as Kiss1. Am J Physiol Metab 2020; 318:E901-19.

[13] Skrapits K, Hrabovszky E. The anatomy of the GnRH neuron network in the human. The GnRH Neuron its Control 2018:149-75.

[14] Silverman JW. The gonadotrophin-releasing hormone(GnRH), neuronal systems: immunocyto-chemistry and in situ hybridization. In: RavenPress E, Knobil E, Neill JD, editors. The physiol-ogy of reproduction; 1994. p. 1683-706.

[15] Schwanzel-Fukuda M, Pfaff DW. Origin of luteinizing hormone-releasing hormone neurons. Nature 1989;338:161-4.

[16] Cho H-J, Shan Y, Whittington NC, et al. Nasal placode development, GnRH neuronal migration and Kallmann syndrome. Front cell Dev Biol 2019;7:121.

[17] Lund C, Yellapragada V, Vuoristo S, et al. Characterization of the human GnRH neuron developmental transcriptome using a GNRH1-TdTomato reporter line in human pluripotent stem cells. DMM Dis Model Mech 2020;13.

[18] Canton APM, Seraphim CE, Brito VN, et al. Pioneering studies on monogenic central precocious puberty. Arch Endocrinol Metab 2019;63:438-44.

[19] Acierno JS, Xu C, Papadakis GE, et al. Pathogenic mosaic variants in congenital hypogonadotropic hypogonadism. Genet Med 2020;22:1759-67.

[20] Messina A, Pulli K, Santini S, et al. Neuron-derived neurotrophic factor is mutated in congenital hypogonadotropic hypogonadism. Am J Hum Genet 2020;106:58-70. 
[21] Smedlund KB, Hill JW. The role of non-neuronal cells in hypogonadotropic hypogonadism. Mol Cell Endocrinol 2020; 518.

[22] Vazquez MJ, Toro CA, Castellano JM, et al. SIRT1 mediates obesity- and nutrient-dependent perturbation of pubertal timing by epigenetically controlling Kiss1 expression. Nat Commun 2018;9.

[23] Roa J, Barroso A, Ruiz-Pino F, et al. Metabolic regulation of female puberty via hypothalamic AMPK-kisspeptin signaling. Proc Natl Acad Sci USA 2018;115:E10758-67.

[24] Talbi R, Navarro VM. Novel insights into the metabolic action of kiss1 neurons. Endocr Connect 2020;9:R124-33.

*[25] Parent AS, Franssen D, Fudvoye J, et al. Developmental variations in environmental influences including endocrine disruptors on pubertal timing and neuroendocrine control: revision of human observations and mechanistic insight from rodents. Front Neuroendocrinol 2015;38:12-36.

[26] Onyango PO, Gesquiere LR, Altmann J, et al. Puberty and dispersal in a wild primate population. Horm Behav 2013;64: $240-9$.

*[27] Biro FM, Pajak A, Wolff MS, et al. Age of menarche in a longitudinal US cohort. J Pediatr Adolesc Gynecol 2018;31: 339-45.

[28] Aksglaede L, Sørensen K, Petersen JH, et al. Recent decline in age at breast development: the Copenhagen puberty study. Pediatrics 2009;123.

[29] Garenne M. Age at menarche in Nigerian demographic surveys. J Biosoc Sci 2020. https://doi.org/10.1017/ S0021932020000504. May 6, 2021.

[30] Pop RM, Tenenboum A, Pop M. Secular trends in height, body mass and mean menarche age in Romanian children and adolescents, 1936-2016. Int J Environ Res Publ Health 2021;18:1-11.

*[31] Eckert-Lind C, Busch AS, Petersen JH, et al. Worldwide secular trends in age at pubertal onset assessed by breast development among girls: a systematic review and meta-analysis. JAMA Pediatr 2020;174.

[32] Ohlsson C, Bygdell M, Celind J, et al. Secular trends in pubertal growth acceleration in Swedish boys born from 1947 to 1996. JAMA Pediatr 2019;173:860-5.

[33] Aksglaede L, Olsen LW, Sørensen TIA, et al. Forty years trends in timing of pubertal growth spurt in 157,000 Danish school children. PloS One 2008;3.

[34] Li W, Liu Q, Deng X, et al. Association between obesity and puberty timing: a systematic review and meta-analysis. Int J Environ Res Publ Health 2017;14.

[35] Soriano-Guillén L, Corripio R, Labarta JI, et al. Central precocious puberty in children living in Spain: incidence, prevalence, and influence of adoption and immigration. J Clin Endocrinol Metab 2010;95:4305-13.

[36] Le Moal J, Rigou A, Le Tertre A, et al. Marked geographic patterns in the incidence of idiopathic central precocious puberty: a nationwide study in France. Eur J Endocrinol 2018;178:33-41.

[37] Kim YJ, Kwon A, Jung MK, et al. Incidence and prevalence of central precocious puberty in Korea: an epidemiologic study based on a national database. J Pediatr 2019;208:221-8.

*[38] Bräuner EV, Busch AS, Eckert-Lind C, et al. Trends in the incidence of central precocious puberty and normal variant puberty among children in Denmark, 1998 to 2017. JAMA Netw open 2020;3:e2015665.

[39] Topor LS, Bowerman K, Machan JT, et al. Central precocious puberty in Boston boys: a 10-year single center experience. PloS One 2018;13.

[40] Fudvoye J, Lopez-Rodriguez D, Franssen D, et al. Endocrine disrupters and possible contribution to pubertal changes. Best Pract Res Clin Endocrinol Metabol 2019;33.

[41] Guth M, Pollock T, Fisher M, et al. Concentrations of urinary parabens and reproductive hormones in girls 6-17 years living in Canada. Int J Hyg Environ Health 2021;231.

[42] Castiello F, Freire C. Exposure to non-persistent pesticides and puberty timing: a systematic review of the epidemiological evidence. Eur J Endocrinol 2021;184:733-49.

[43] Sferruzzi-Perri AN, Vaughan OR, Forhead AJ, et al. Hormonal and nutritional drivers of intrauterine growth. Curr Opin Clin Nutr Metab Care 2013;16:298-309.

[44] Street ME, Bernasconi S. Endocrine-disrupting chemicals in human fetal growth. Int J Mol Sci 2020;21.

[45] Roncati L, Piscioli F, Pusiol T. The endocrine disruptors among the environmental risk factors for stillbirth. Sci Total Environ 2016;563-564:1086-7.

[46] Vizcaino E, Grimalt JO, Fernández-Somoano A, et al. Transport of persistent organic pollutants across the human placenta. Environ Int 2014;65:107-15.

[47] Balakrishnan B, Henare K, Thorstensen EB, et al. Transfer of bisphenol A across the human placenta. Am J Obstet Gynecol 2010;202. 393.e1-7.

[48] Bradman A, Barr DB, Claus Henn BG, et al. Measurement of pesticides and other toxicants in amniotic fluid as a potential biomarker of prenatal exposure: a validation study. Environ Health Perspect 2003;111:1779-82.

[49] Benedikt W, Karin P, Pius M, et al. Transfer and metabolism of the xenoestrogen zearalenone in human perfused placenta. Environ Health Perspect 2021;127:107004.

[50] Yang C, Song G, Lim W. A mechanism for the effect of endocrine disrupting chemicals on placentation. Chemosphere 2019;231:326-36.

[51] Zong T, Lai L, Hu J, et al. Maternal exposure to di-(2-ethylhexyl) phthalate disrupts placental growth and development in pregnant mice. J Hazard Mater 2015;297:25-33.

*[52] Gore AC, Chappell VA, Fenton SE, et al. EDC-2: the endocrine society's second scientific statement on endocrinedisrupting chemicals. Endocr Rev 2015;36:E1-150.

[53] Yoshida K, Tobet SA, Crandall JE, et al. The migration of luteinizing hormone-releasing hormone neurons in the developing rat is associated with a transient, caudal projection of the vomeronasal nerve. J Neurosci 1995;15: 7769-77.

[54] Dodé C, Hardelin J-P. Kallmann syndrome. Eur J Hum Genet 2009;17:139-46.

[55] Vosges M, Le Page Y, Chung B-C, et al. 17alpha-ethinylestradiol disrupts the ontogeny of the forebrain GnRH system and the expression of brain aromatase during early development of zebrafish. Aquat Toxicol 2010;99:479-91. 
[56] Vosges M, Kah O, Hinfray N, et al. 17 $\alpha$-Ethinylestradiol and nonylphenol affect the development of forebrain GnRH neurons through an estrogen receptors-dependent pathway. Reprod Toxicol 2012;33:198-204.

[57] Pillon D, Cadiou V, Angulo L, et al. Maternal exposure to 17-alpha-ethinylestradiol alters embryonic development of GnRH-1 neurons in mouse. Brain Res 2012;1433:29-37.

[58] Bai Y, Chang F, Zhou R, et al. Increase of anteroventral periventricular kisspeptin neurons and generation of E2induced LH-surge system in male rats exposed perinatally to environmental dose of bisphenol-A. Endocrinology 2011;152:1562-71.

[59] Biehl MJ, Raetzman LT. Developmental origins of hypothalamic cells controlling reproduction. Semin Reprod Med 2017;35:121-9.

[60] García-Galiano D, Pinilla L, Tena-Sempere M. Sex steroids and the control of the Kiss1 system: developmental roles and major regulatory actions. J Neuroendocrinol 2012;24:22-33.

[61] Stévant I, Nef S. Genetic control of gonadal sex determination and development. Trends Genet 2019;35:346-58.

[62] Frye CA. Endocrine-Disrupting chemicals. Elucidating our understanding of their role in sex and gender-relevant end points. In: Vitamins and hormones. Academic Press Inc.; 2014. p. 41-98.

[63] Holm JB, Mazaud-Guittot S, Banhos Danneskiold-Sams N, et al. Intrauterine exposure to paracetamol and aniline impairs female reproductive development by reducing follicle reserves and fertility. ToxSci Adv 2016:1-32.

[64] Lawson C, Gieske M, Murdoch B, et al. Gene expression in the fetal mouse ovary is altered by exposure to low doses of bisphenol A. Biol Reprod 2011;84:79-86.

[65] Zhang XF, Zhang T, Han Z, et al. Transgenerational inheritance of ovarian development deficiency induced by maternal diethylhexyl phthalate exposure. Reprod Fertil Dev 2015;27:1213-21.

*[66] Johansson HKL, Svingen T, Fowler PA, et al. Environmental influences on ovarian dysgenesis-developmental windows sensitive to chemical exposures. Nat Rev Endocrinol 2017;13:400-14.

[67] Hu J, Du G, Zhang W, et al. Short-term neonatal/prepubertal exposure of dibutyl phthalate (DBP) advanced pubertal timing and affected hypothalamic kisspeptin/GPR54 expression differently in female rats. Toxicology 2013;314:65-75.

[68] Yang R, Wang Y-M, Zhang L, et al. Prepubertal exposure to an oestrogenic mycotoxin zearalenone induces central precocious puberty in immature female rats through the mechanism of premature activation of hypothalamic kisspeptin-GPR54 signaling. Mol Cell Endocrinol 2016;437:62-74.

[69] Kurian JR, Keen KL, Kenealy BP, et al. Acute influences of bisphenol a exposure on hypothalamic release of gonadotropin-releasing hormone and kisspeptin in female rhesus monkeys. Endocrinology 2015;156:2563-70.

[70] Devillers MM, Petit F, Giton F, et al. Age-dependent vulnerability of the ovary to AhR-mediated TCDD action before puberty: evidence from mouse models. Chemosphere 2020;258:127361.

[71] Desaulniers D, Xiao G-H, Leingartner K, et al. Comparisons of brain, uterus, and liver mRNA expression for cytochrome p450s, DNA methyltransferase-1, and catechol-o-methyltransferase in prepubertal female Sprague-Dawley rats exposed to a mixture of aryl hydrocarbon receptor agonists. Toxicol Sci 2005;86:175-84.

[72] Balaguer P, Delfosse V, Bourguet W. Mechanisms of endocrine disruption through nuclear receptors and related pathways. Curr Opin Endocr Metab Res 2019;7:1-8.

[73] Klenke U, Constantin S, Wray S. BPA directly decreases GnRH neuronal activity via noncanonical pathway. Endocrinology 2016;157:1980-90.

[74] Tohme M, Prud'homme SM, Boulahtouf A, et al. Estrogen-related receptor gamma is an in vivo receptor of bisphenol A. FASEB J Off Publ Fed Am Soc Exp Biol 2014;28:3124-33.

[75] Mimura J, Fujii-Kuriyama Y. Functional role of AhR in the expression of toxic effects by TCDD in Biochimica et biophysica acta - general subjects. Biochim Biophys Acta; 2003. p. 263-8.

[76] Raggi F, Russo D, Urbani C, et al. Divergent effects of dioxin- or non- dioxin- like polychlorinated biphenyls on the apoptosis of primary cell culture from the mouse dioxin pituitary gland. PloS One 2016;11.

[77] Yueh M-F, Huang Y-H, Hiller A, et al. Involvement of the xenobiotic response element (XRE) in Ah receptor-mediated induction of human UDP-glucuronosyltransferase 1A1. J Biol Chem 2003;278:15001-6.

[78] Thomas P, Dong J. Binding and activation of the seven-transmembrane estrogen receptor GPR30 by environmental estrogens: a potential novel mechanism of endocrine disruption. J Steroid Biochem Mol Biol 2006;102:175-9.

[79] Murata M, Kang J-H. Bisphenol A (BPA) and cell signaling pathways. Biotechnol Adv 2018;36:311-27.

[80] Zhuang W, Wu K, Wang Y, et al. Association of serum bisphenol-a concentration and male reproductive function among exposed workers. Arch Environ Contam Toxicol 2015;68:38-45.

[81] Loffredo LF, Berdnikovs MECS. Endocrine disruptor bisphenol a (BPA) triggers systemic para-inflammation and is sufficient to induce airway allergic sensitization in mice. Nutrients 2020;12.

[82] Rock KD, Patisaul HB. Environmental mechanisms of neurodevelopmental toxicity. Curr Environ Heal reports 2018;5: $145-57$.

[83] Lopez-Rodriguez D, Franssen D, Bakker J, et al. Cellular and molecular features of EDC exposure: consequences for the GnRH network. Nat Rev Endocrinol 2021;17:83-96.

[84] Lehle JD, McCarrey JR. Differential susceptibility to endocrine disruptor-induced epimutagenesis. Environ Epigenetics 2020;6.

[85] Van Cauwenbergh O, Di Serafino A, Tytgat J, et al. Transgenerational epigenetic effects from male exposure to endocrine-disrupting compounds: a systematic review on research in mammals. Clin Epigenet 2020;12.

[86] Rattan S, Beers HK, Kannan A, et al. Prenatal and ancestral exposure to di(2-ethylhexyl) phthalate alters gene expression and DNA methylation in mouse ovaries. Toxicol Appl Pharmacol 2019;379:114629.

[87] Palanivel TM, Sivakumar N, Al-Ansari A, et al. Bioremediation of copper by active cells of Pseudomonas stutzeri LA3 isolated from an abandoned copper mine soil. J Environ Manag 2020;253:109706.

[88] Huang A, Reinehr T, Roth CL. Connections between obesity and puberty. Curr Opin Endocr Metab Res 2020;14:160-8.

*[89] Lee JE, Jung HW, Lee YJ, et al. Early-life exposure to endocrine-disrupting chemicals and pubertal development in girls. Ann Pediatr Endocrinol Metab 2019;24:78-91.

[90] Aylwin CF, Vigh-Conrad K, Lomniczi A, et al. The emerging role of chromatin remodeling factors in female pubertal development. Neuroendocrinology 2019;109:208-17. 
[91] Toro CA, Wright H, Aylwin CF, et al. Trithorax dependent changes in chromatin landscape at enhancer and promoter regions drive female puberty. Nat Commun 2018;9:57.

[92] Tomikawa J, Uenoyama Y, Ozawa M, et al. Epigenetic regulation of Kiss1 gene expression mediating estrogen-positive feedback action in the mouse brain. Proc Natl Acad Sci USA 2012;109:E1294-301.

[93] Messina A, Langlet F, Chachlaki K, et al. A microRNA switch regulates the rise in hypothalamic GnRH production before puberty. Nat Neurosci 2016;19:835-44.

[94] Jones PA, Takai D. The role of DNA methylation in mammalian epigenetics. Science 2001;293:1068-70.

[95] Yang C, Ye J, Li X, et al. DNA methylation patterns in the hypothalamus of female pubertal goats. PloS One 2016;11: e0165327.

*[96] McCarthy MM, Nugent BM. Epigenetic contributions to hormonally-mediated sexual differentiation of the brain. J Neuroendocrinol 2013;25:1133-40.

[97] Kurian JR, Keen KL, Terasawa E. Epigenetic changes coincide with in vitro primate GnRH neuronal maturation. Endocrinology 2010;151:5359-68.

[98] Almstrup K, Frederiksen H, Andersson AM, et al. Levels of endocrine-disrupting chemicals are associated with changes in the peri-pubertal epigenome. Endocr Connect 2020;9:845-57.

[99] Almstrup K, Lindhardt Johansen M, Busch AS, et al. Pubertal development in healthy children is mirrored by DNA methylation patterns in peripheral blood. Sci Rep 2016;6.

[100] Kundakovic M, Gudsnuk K, Franks B, et al. Sex-specific epigenetic disruption and behavioral changes following lowdose in utero bisphenol A exposure. Proc Natl Acad Sci USA 2013;110:9956-61.

[101] Cheong A, Johnson SA, Howald EC, et al. Gene expression and DNA methylation changes in the hypothalamus and hippocampus of adult rats developmentally exposed to bisphenol A or ethinyl estradiol: a CLARITY-BPA consortium study. Epigenetics 2018;13:704-20.

[102] Ye X, Zhou X, Hennings R, et al. Potential external contamination with bisphenol A and other ubiquitous organic environmental chemicals during biomonitoring analysis: an elusive laboratory challenge. Environ Health Perspect $2013 ; 121$.

[103] Malloy MA, Kochmanski JJ, Jones TR, et al. Perinatal bisphenol A exposure and reprogramming of imprinted gene expression in the adult mouse brain. Front Genet 2019;10:951.

[104] Kumar D, Thakur MK. Effect of perinatal exposure to Bisphenol-A on DNA methylation and histone acetylation in cerebral cortex and hippocampus of postnatal male mice. J Toxicol Sci 2017;42:281-9.

*[105] Mueller JK, Heger S. Endocrine disrupting chemicals affect the gonadotropin releasing hormone neuronal network. Reprod Toxicol 2014;44:73-84.

[106] Gore AC, Walker DM, Zama AM, et al. Early life exposure to endocrine-disrupting chemicals causes lifelong molecular reprogramming of the hypothalamus and premature reproductive aging. Mol Endocrinol 2011;25:2157-68.

[107] Stouder C, Paoloni-Giacobino A. Specific transgenerational imprinting effects of the endocrine disruptor methoxychlor on male gametes. Reproduction 2011;141:207-16.

[108] Topper VY, Walker DM, Gore AC. Sexually dimorphic effects of gestational endocrine-disrupting chemicals on microRNA expression in the developing rat hypothalamus. Mol Cell Endocrinol 2015;414:42-52.

[109] Sangiao-Alvarellos S, Manfredi-Lozano M, Ruiz-Pino F, et al. Changes in hypothalamic expression of the Lin28/let-7 system and related microRNAs during postnatal maturation and after experimental manipulations of puberty. Endocrinology 2013;154:942-55.

[110] Veiga-Lopez A, Luense LJ, Christenson LK, et al. Developmental programming: gestational bisphenol-a treatment alters trajectory of fetal ovarian gene expression. Endocrinology 2013;154:1873-84.

[111] Gao G-Z, Zhao Y, Li H-X, et al. Bisphenol A-elicited miR-146a-5p impairs murine testicular steroidogenesis through negative regulation of Mta3 signaling. Biochem Biophys Res Commun 2018;501:478-85.

[112] Manikkam M, Tracey R, Guerrero-Bosagna C, et al. Dioxin (TCDD) induces epigenetic transgenerational inheritance of adult onset disease and sperm epimutations. PloS One 2012;7:e46249.

[113] Manikkam M, Guerrero-Bosagna C, Tracey R, et al. Transgenerational actions of environmental compounds on reproductive disease and identification of epigenetic biomarkers of ancestral exposures. PloS One 2012;7:e31901.

[114] Geoffron S, Abi Habib W, Chantot-Bastaraud S, et al. Chromosome 14q32.2 imprinted region disruption as an alternative molecular diagnosis of Silver-Russell syndrome. J Clin Endocrinol Metab 2018;103:2436-46.

[115] Drobna Z, Henriksen AD, Wolstenholme JT, et al. Transgenerational effects of bisphenol A on gene expression and DNA methylation of imprinted genes in brain. Endocrinology 2018;159:132-44. 